\title{
Rapport des rencontres EAP et du 23e congrès EAP à Belgrade
}

Peter Schulthess

Les rencontres d'automne de l'EAP ont eu lieu du 18 au 20 octobre 2018 à Belgrade. Le 23e Congrès spécialisé de l'EAP a eu lieu en parallèle du 18 au 21 octobre, il fut simultanément le $8 \mathrm{e}$ Congrès de l'Association Serbe de Psychothérapie.

\section{L’EAP a un nouveau Président}

Charles Cassar en provenance de Malte a repris, en tant que vice-président de façon statutaire, un peu plus tôt que prévu la fonction de Président, après que l'actuel Président, Philippe Vranken originaire de Belgique, ait renoncé à son poste début mai en raison d'un conflit avec le Conseil d'administration. Son mandat aurait dû durer encore jusqu'à février 2019. Il en a fait part dans une lettre adressée aux membres du Conseil d'administration élargi, sans que la raison réelle du conflit en ressorte. Des craintes selon lesquelles une partie du Conseil exécutif ne voulait pas le suivre sur le plan politique dans la mise en œuvre des décisions du Conseil d'administration élargi, également sur le plan du lobbying, se sont révélées infondées. En dépit des discussions qui se sont tenues au début de la rencontre de Belgrade, les raisons du retrait en conflit restent floues.

Philippe Vranken fut un président très proactif, qui a initié de nombreux changements dans l'EAP et nombreux étaient ceux qui le considéraient comme un porteur d'espoir. Les représentants des anciennes structures du pouvoir ne le voyaient pas d'un très bon œil et ont essayé d'entraver le président.

Même s'il se retire prématurément, la dynamisation de l'EAP lui revient.

Charles Cassar a l'intention de continuer à faire avancer les réformes et activités lancées par Philippe Vranken et notamment sur la scène européenne, à continuer à être politiquement actif pour notre profession. Il a pour mérite la promulgation à Malte d'une loi sur la psychothérapie qui ne considère pas la profession de psychothérapeute comme une profession psychologique ou médicale, mais comme un métier scientifique indépendant en toute autonomie.

\section{Nouvelle loi sur la psychothérapie à Malte et en Croatie}

Au fil des années, des lois régissant la psychothérapie comme une profession indépendante sont entrées en vigueur dans les deux pays. C'est un progrès et un succès importants pour l'EAP aussi. Une réforme dans le secteur de la formation des hautes écoles y est associée. Il est désormais non seulement possible d'obtenir un diplôme universitaire sous la forme d'un Master ou d'un $\mathrm{PhD}$ en psychothérapie, mais les établissements de formation postgraduée ont la possibilité de se faire reconnaitre en tant que hautes écoles de pratique (ce qui correspond chez nous aux écoles techniques) et de proposer à la fois des programmes de master et de doctorat. À l'avenir, il sera ainsi possible à Malte d'obtenir un master en Gestalt-thérapie ou un $\mathrm{PhD}$ en Gestalt-thérapie, dans la mesure l'Institut local en Gestalt-thérapie a reçu la reconnaissance d'école technique. De tels programmes (pour différentes orientations thérapeutiques) existent depuis longtemps dans l'espace anglo-saxon sous la forme de projets de coopération d'instituts privés de formation postgraduée avec des universités.

\section{Comptes-rendus de colloque}

Le Président du Comité pour la Science et la Recherche (SARC), Peter Schulthess, a fait un compte-rendu des trois congrès scientifiques auxquels s'est rendu le SARC cette année. Lors du congrès de l'Association européenne de Psychiatrie (EPA), il s'est avéré que la psychothérapie et la recherche en psychothérapie n'ont guère suscité le débat. Les présentations ont concerné des sujets psychiatriques, neuroscientifiques et pharmacologiques. Le Conseil d'administration de l'EPA s'intéresse toutefois à faire connaitre davantage la psychothérapie à ses membres et recherche la coopération et la participation de l'EAP pendant les congrès.

La revue Psychotherapie-Wissenschaft, cahier 2-2018, a rendu compte des congrès de la Fédération Internationale de Psychothérapie (IFP) et de la Société pour la Recherche en Psychothérapie (SPR) de façon approfondie. Des conclusions choisies sur l'état de la discussion ont également été transmises au Conseil d'administration. Les membres du Conseil d'administration élargi de l'EAP ont exprimé leur reconnaissance pour ces rapports. Il a été souhaité que les données de congrès de l'EAP soient mises à disposition sur le site Internet (pas seulement les propres données), tout comme des liens vers des revues majeures.

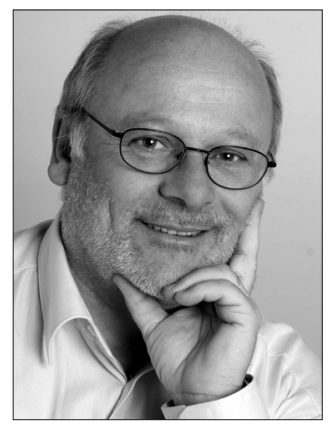


Nouveau secrétariat et nouveau comité des finances

Le bureau de l'EAP a déménagé pour des raisons de coût. Il se trouve désormais dans la Mariahilfer Strasse 1d/3e étage/porte 13, 1060 Vienne. Les finances sont un sujet brûlant à l'EAP : les recettes diminuent, les dépenses augmentent. Il est nécessaire de le maitriser en créant des possibilités de recettes supplémentaires et en abaissant les coûts. Un groupe de travail vient dêtre lancé, devant agir comme un comité des finances et qui approfondit des idées correspondantes, s'exprime en tant qu'organe consultatif du trésorier et du Comité exécutif.

\section{Chambre des établissements}

de formation postgraduée

Pour la seconde fois a eu également lieu une réunion des représentants des établissements de formation postgraduée avec accréditation EAP. Cela a permis au SARC de présenter une nouvelle fois l'étude SPRISTAD de la SPR et d'inciter les établissements de formation postgraduée à participer. La SPRISTAD analyse le développement professionnel et personnel des participantes et participants à la formation postgraduée pendant le déroulement de leur formation. Cela a permis par ailleurs de s'intéresser au perfectionnement des programmes de sorte qu'ils atteignent le niveau de formation 7 (correspond à un Master). Pour ce faire, il est nécessaire qu'un module de recherche fasse également partie des programmes de formation postgraduée. Il faut continuer à approfondir ce sujet lors des prochaines réunions avec une manifestation d'une journée. Dans la révision en cours des statuts, les établissements de formation postgraduée devraient obtenir une chambre personnelle.
Document sur le Brexit du point de vue des psychothérapeutes

Sur demande de la Présidente de l'Association britannique de psychothérapie (UKCP), les intéressés se sont réunis lors d'une réunion séparée afin d'échanger sur le Brexit à venir et ses effets sur les psychothérapeutes, la formation et les patientes et patients. Des aspects ont été réunis qui doivent être ultérieurement compilés lors des réunions en un seul document qui devrait être aussi mis à disposition politiquement au niveau de l'Union européenne et des parlementaires britanniques.

\section{Colloque réussi}

Près de 500 participants sont venus au congrès mentionné au début et qui avait lieu en parallèle. Le sujet du colloque était: "Sense and Sensitivity in Psychotherapie». De nombreux exposés techniques et ateliers en serbe et en anglais ont été proposés. Les congrès de l'EAP donnent toujours loccasion à l'association nationale organisatrice de faire connaitre l'implication européenne de l'association nationale pour ses membres, en invitant des intervenantes et intervenants des instances de l'EAP. À l'inverse, l'EAP peut se présenter aux membres d'une association nationale avec ses réalisations pour la profession de psychothérapie. Le SARC a aussi utilisé lopportunité de rendre compte, par l'intermédiaire de "Sense and Sensitivity in Psychotherapiy Research », afin d'inciter les praticiennes et praticiens à la participation.

Peter Schulthess est membre du Conseil d'administration de l'ASP et la représente en collaboration avec Gabriela Rüttimann dans l'EAP. 